Article

\title{
Some Results of Fekete-Szegö Type. Results for Some Holomorphic Functions of Several Complex Variables
}

\author{
Renata Długosz ${ }^{1, *(1)}$ and Piotr Liczberski ${ }^{2}$ (I) \\ 1 Centre of Mathematics and Physics, Lodz University of Technology, Ul. Żwirki 36, 90-924 Łódź, Poland \\ 2 Institute of Mathematics, Lodz University of Technology, Ul. Żwirki 36, 90-924 Łódź, Poland; \\ piotr.liczberski@p.lodz.pl \\ * Correspondence: renata.dlugosz@p.lodz.pl
}

Received: 29 August 2020; Accepted: 9 October 2020; Published: 16 October 2020

check for updates

\begin{abstract}
This paper is devoted to a generalization of the well-known Fekete-Szegö type coefficients problem for holomorphic functions of a complex variable onto holomorphic functions of several variables. The considerations concern three families of such functions $f$, which are bounded, having positive real part and which Temljakov transform $L f$ has positive real part, respectively. The main result arise some sharp estimates of the Minkowski balance of a combination of 2-homogeneous and the square of 1-homogeneous polynomials occurred in power series expansion of functions from aforementioned families.
\end{abstract}

Keywords: holomorphic functions of scv; $n$-circular domains in $\mathbb{C}^{n \%}$; minkowski function; fekete-Szegö type estimates

MSC2010: 32A30; 30C45

\section{Introduction}

Since the several complex variables geometric analysis depends on the type of domains in $\mathbb{C}^{n}$ (see for instance References [1-3]), we consider a special, but wide class of domains in $\mathbb{C}^{n}$. We say that a domain $\mathcal{G} \subset \mathbb{C}^{n}, n \geq 1$, is complete $n$-circular if $z \lambda=\left(z_{1} \lambda_{1}, \ldots, z_{n} \lambda_{n}\right) \in \mathcal{G}$ for each $z=\left(z_{1}, \ldots, z_{n}\right) \in \mathcal{G}$ and every $\lambda=\left(\lambda_{1}, \ldots, \lambda_{n}\right) \in \overline{U^{n}}$, where $U^{n}$ is the open unit polydisc in $\mathbb{C}^{n}$, that is, the product of $n$ copies of the open unit disc $U=\{\zeta \in \mathbb{C}:|\zeta|<1\}$. From now on by $\mathcal{G}$ will be denoted a bounded complete $n$-circular domain in $\mathbb{C}^{n}, n \geq 1$. Such bounded domain $\mathcal{G}$ and its boundary $\partial \mathcal{G}$ can be redefined as follows

$$
\mathcal{G}=\left\{z \in \mathbb{C}^{n}: \mu_{\mathcal{G}}(z)<1\right\}, \partial \mathcal{G}=\left\{z \in \mathbb{C}^{n}: \mu_{\mathcal{G}}(z)=1\right\},
$$

using the Minkowski function $\mu_{\mathcal{G}}: \mathbb{C}^{n} \rightarrow[0, \infty)$

$$
\mu_{\mathcal{G}}(z)=\inf \left\{t>0: \frac{1}{t} z \in \mathcal{G}\right\}, z \in \mathbb{C}^{n} .
$$

It is well-known (see e.g, Reference [4]) that $\mu_{\mathcal{G}}$ is a norm in $\mathbb{C}^{n}$ if $\mathcal{G}$ is a convex bounded complete $n$-circular domain. 
The function $\mu_{\mathcal{G}}$ is very useful in research the space $\mathcal{H}_{\mathcal{G}}$ of holomorphic functions $f: \mathcal{G} \rightarrow \mathbb{C}$. By $\mathcal{H}_{\mathcal{G}}(1)$ will be denoted the collection of all $f \in \mathcal{H}_{\mathcal{G}}$, normalized by the condition $f(0)=1$. In the paper we consider the following subfamilies of $\mathcal{H}_{\mathcal{G}}$

$$
\begin{aligned}
& \mathcal{B}_{\mathcal{G}}=\left\{f \in \mathcal{H}_{\mathcal{G}}:|f(z)|<1, z \in \mathcal{G}\right\}, \\
& \mathcal{C}_{\mathcal{G}}=\left\{f \in \mathcal{H}_{\mathcal{G}}(1): \operatorname{Re} f(z)>0, z \in \mathcal{G}\right\}, \\
& \mathcal{V}_{\mathcal{G}}=\left\{f \in \mathcal{H}_{\mathcal{G}}(1): \operatorname{Re} \mathcal{L} f(z)>0, z \in \mathcal{G}\right\},
\end{aligned}
$$

where $\mathcal{L}: \mathcal{H}_{\mathcal{G}} \longrightarrow \mathcal{H}_{\mathcal{G}}$ means the Temljakov [5] linear operator

$$
\mathcal{L} f(z)=f(z)+D f(z)(z), z \in \mathcal{G}
$$

defined by the Frechet differential $D f(z)$ of $f$ at the point $z$. Note that the operator $\mathcal{L}$ is invertible and its inverse has the form

$$
\mathcal{L}^{-1} f(z)=\int_{0}^{1} f(z t) d t, z \in \mathcal{G} .
$$

Let us recall that every function $f \in \mathcal{H}_{\mathcal{G}}$ has a unique power series expansion

$$
f(z)=\sum_{m=0}^{\infty} Q_{f, m}(z), z \in \mathcal{G},
$$

where $Q_{f, m}: \mathbb{C}^{n} \rightarrow \mathbb{C}, m \in \mathbb{N} \cup\{0\}$, are $m$-homogeneous polynomials. Usually the notion of $m$-homogeneous polynomial $Q_{m}: \mathbb{C}^{n} \longrightarrow \mathbb{C}$ is defined by the formula

$$
Q_{m}(z)=L_{m}\left(z^{m}\right)=L_{m}(z, \ldots, z), z \in \mathbb{C}^{n},
$$

where $L_{m}:\left(\mathbb{C}^{n}\right)^{m} \longrightarrow \mathbb{C}$ is an $m$-linear mapping (0-homogeneous polynomial means a constant function $Q_{0}: \mathbb{C}^{n} \rightarrow \mathbb{C}$ ). Note that the homogeneous polynomials occured in the expansion (1) have the form

$$
Q_{f, m}(z)=\frac{1}{m !} D^{m} f(0)\left(z^{m}\right) .
$$

A simple kind of 1-homogeneous polynomial is the following linear functional $J \in\left(\mathbb{C}^{n}\right)^{*}$

$$
J(z)=\sum_{j=1}^{n} z_{j}, z=\left(z_{1}, \ldots, z_{n}\right) \in \mathbb{C}^{n}
$$

We will use the following generalization of the notion of the norm of $m$-homogeneous polynomial $Q_{m}: \mathbb{C}^{n} \rightarrow \mathbb{C}$, that is, the $\mu_{\mathcal{G}}$-balance of $Q_{m}[6-8]$

$$
\mu_{\mathcal{G}}\left(Q_{m}\right)=\sup _{w \in \mathbb{C}^{n} \backslash\{0\}} \frac{\left|Q_{m}(w)\right|}{\left(\mu_{G}(w)\right)^{m}}=\sup _{v \in \partial \mathcal{G}}\left|Q_{m}(v)\right|=\sup _{u \in \mathcal{G}}\left|Q_{m}(u)\right|,
$$

which is identical with the norm $\left\|Q_{m}\right\|$ if $\mathcal{G}$ is convex. The notion $\mu_{\mathcal{G}}$-balance of $m$-homogeneous polynomial brings a very useful inequality

$$
\left|Q_{m}(z)\right| \leq \mu_{\mathcal{G}}\left(Q_{m}\right)\left(\mu_{G}(z)\right)^{m},
$$


which generalize the well-known inequality

$$
\left|Q_{m}(z)\right| \leq\left\|Q_{m}\right\|\|z\|^{m} .
$$

Let us denote by $I$ the linear functional

$$
I=\left(\mu_{\mathcal{G}}(J)\right)^{-1} J
$$

and by $I^{m}, m \geq 1$, the $m$-homogeneous polynomial $I^{m}: \mathbb{C}^{n} \rightarrow \mathbb{C}$

$$
I^{m}(z)=(I(z))^{m}, z \in \mathbb{C}^{n} .
$$

It is obvious that $\mu_{\mathcal{G}}\left(I^{m}\right)=1$.

In many papers (see for instance References [9-13]) there are presented some sharp estimations of $m$-homogeneous polynomials $Q_{f, m}, m \geq 1$, for functions $f$ of the form (1) from different subfamilies of $\mathcal{H}_{\mathcal{G}}$. Below we give three Bavrin's [9] estimates, in the case $\mathbb{C}^{n}, n \geq 1$, in term of $\mu_{\mathcal{G}}$-balances of $m$-homogeneous polynomials, $m \geq 1$

$$
\mu_{\mathcal{G}}\left(Q_{f, m}\right) \leq\left\{\begin{array}{c}
1, \text { for } f \in \mathcal{B}_{\mathcal{G}} \\
2, \text { for } f \in \mathcal{C}_{\mathcal{G}} \\
\frac{2}{m+1}, \text { for } f \in \mathcal{V}_{\mathcal{G}}
\end{array}, m \geq 1\right.
$$

\section{Main Results}

In the present paper we give for $f \in \mathcal{B}_{\mathcal{G}}(0)=\left\{f \in \mathcal{B}_{\mathcal{G}}: f(0)=0\right\}$ (also for $f \in \mathcal{C}_{\mathcal{G}}$ and $f \in \mathcal{V}_{\mathcal{G}}$ ) a kind sharp estimate for the pair of homogeneous polynomials $Q_{f, 2}, Q_{f, 1}$, that is, sharp estimate

$$
\mu_{\mathcal{G}}\left(Q_{f, 2}-\lambda\left(Q_{f, 1}\right)^{2}\right) \leq M(\lambda), \lambda \in \mathbb{C} .
$$

It is a generalization of a solution of the well known Fekete-Szegö coefficient problem in complex plane [14] onto the case of several complex variables. The first result we demonstrate in the following theorem, which is a generalization of a result of Keogh and Merkes [15]:

Theorem 1. Let $\varphi \in \mathcal{B}_{\mathcal{G}}(0)$ be a function of the form

$$
\varphi(z)=\sum_{m=1}^{\infty} Q_{\varphi, m}(z), z \in \mathcal{G}
$$

Then, for every $\gamma \in \mathbb{C}$ there holds the sharp estimate

$$
\mu_{\mathcal{G}}\left(Q_{\varphi, 2}-\gamma\left(Q_{\varphi, 1}\right)^{2}\right) \leq \max \{1,|\gamma|\}
$$

Proof. Let us fix arbitrarily $z \in \mathcal{G} \backslash\{0\}$. Then using the classic Schwarz Lemma to the function $U \ni \zeta \rightarrow$ $\varphi\left(\zeta \frac{z}{\mu_{\mathcal{G}}(z)}\right) \in U$ (at the point $\zeta=\mu_{\mathcal{G}}(z) \in U$ ), we obtain the inequality

$$
|\varphi(z)| \leq \mu_{\mathcal{G}}(z), z \in \mathcal{G} \backslash\{0\}
$$

(it is also true for $z=0$ ). 
Now, by this result we see that for every $z \in \mathcal{G}$, the function

$$
\Phi(\zeta)=\left\{\begin{array}{c}
\frac{\varphi(\zeta z)}{\zeta}, \zeta \in U \backslash\{0\} \\
\lim _{\zeta \rightarrow 0} \frac{\varphi(\zeta z)}{\zeta}, \zeta=0
\end{array}\right.
$$

transforms holomorphically the disc $U$ into itself, fixes the point $\zeta=0$ and has the expression

$$
\Phi(\zeta)=\sum_{m=0}^{\infty} \beta_{m} \zeta^{m}, \zeta \in U,
$$

where $\beta_{m}=Q_{\varphi, m+1}(z)$, for nonegative integers $m$.

Thus, in view of the well known $[16,17]$ sharp coefficient estimates

$$
\begin{aligned}
& \left|\beta_{m}\right| \leq 1, m=0,1, \ldots \\
& \left|\beta_{1}\right| \leq 1-\left|\beta_{0}\right|^{2}
\end{aligned}
$$

we obtain for every $z \in \mathcal{G}$

$$
\begin{gathered}
\left|Q_{\varphi, m}(z)\right| \leq 1, m=1,2, \ldots \\
\left|Q_{\varphi, 2}(z)\right| \leq 1-\left|Q_{\varphi, 1}(z)\right|^{2} .
\end{gathered}
$$

Therefore, for $z \in \mathcal{G}$ and every $\gamma \in \mathbb{C}$

$$
\begin{gathered}
\left|Q_{\varphi, 2}(z)-\gamma\left(Q_{\varphi, 1}(z)\right)^{2}\right| \leq\left|Q_{\varphi, 2}(z)\right|+|\gamma|\left|Q_{\varphi, 1}(z)\right|^{2} \leq 1-\left|Q_{\varphi, 1}(z)\right|^{2}+|\gamma|\left|Q_{\varphi, 1}(z)\right|^{2} \\
=1+(|\gamma|-1)\left|Q_{\varphi, 1}(z)\right|^{2} \leq \max \{1,|\gamma|\}
\end{gathered}
$$

because $(|\gamma|-1)\left|Q_{\varphi, 1}(z)\right|^{2} \leq 0$ if $|\gamma|<1$ and $0 \leq(|\gamma|-1)\left|Q_{\varphi, 1}(z)\right|^{2} \leq|\gamma|-1$ if $|\gamma| \geq 1$.

Consequently,

$$
\sup _{z \in \mathcal{G}}\left|Q_{\varphi, 2}(z)-\gamma\left(Q_{\varphi, 1}(z)\right)^{2}\right| \leq \max \{1,|\gamma|\}
$$

The above inequality gives the estimate (4) from the thesis by the definition of $\mu_{\mathcal{G}}$-balance of homogeneous polynomials and the fact that $Q_{\varphi, 2}-\gamma\left(Q_{\varphi, 1}\right)^{2}$ is a 2-homogeneous polynomial.

It remains the problem of the sharpness of the estimation (4). First, we prove that in the case $|\gamma| \geq 1$, the equality in $(4)$ is attained by the function $\widetilde{\varphi} \in \mathcal{B}_{\mathcal{G}}(0)$

$$
\widetilde{\varphi}(z)=I(z), z \in \mathcal{G} .
$$

Indeed, since $Q_{\widetilde{\varphi}, 1}=I, Q_{\widetilde{\varphi}, 2}=0$ and $\mu_{\mathcal{G}}\left(I^{2}\right)=1$, we have

$$
\mu_{\mathcal{G}}\left(Q_{\widetilde{\varphi}, 2}-\gamma\left(Q_{\widetilde{\varphi}, 1}\right)^{2}\right)=\mu_{\mathcal{G}}\left(-\gamma\left(Q_{\widetilde{\varphi}, 1}\right)^{2}\right)=|\gamma| \mu_{\mathcal{G}}\left(\left(Q_{\widetilde{\varphi}, 1}\right)^{2}\right)=|\gamma|=\max \{1,|\gamma|\} .
$$

Now, we show that in the case $|\gamma|<1$ the equality in (4) realizes the function $\widehat{\varphi} \in \mathcal{B}_{\mathcal{G}}(0)$

$$
\widehat{\varphi}(z)=I^{2}(z), z \in \mathcal{G} \text {. }
$$


Indeed, since $Q_{\widehat{\varphi}, 1}=0, Q_{\widehat{\varphi}, 2}=I^{2}$, we get

$$
\mu_{\mathcal{G}}\left(Q_{\widehat{\varphi}, 2}-\gamma\left(Q_{\widehat{\varphi}, 1}\right)^{2}\right)=\mu_{\mathcal{G}}\left(Q_{\widehat{\varphi}, 2}\right)=1=\max \{1,|\gamma|\} .
$$

This completes the proof.

A next theorem includes a solution of the Fekete-Szegö type problem in the family $\mathcal{C}_{\mathcal{G}}$.

Theorem 2. Let $\mathcal{G} \subset \mathbb{C}^{n}$ be a bounded complete n-circular domain and let $p \in \mathcal{C}_{\mathcal{G}}$. If the expansion of the function $p$ into a series of $m$-homogenous polynomials $Q_{p, m}$ has the form

$$
p(z)=1+\sum_{m=1}^{\infty} Q_{p, m}(z), z \in \mathcal{G},
$$

then for the homogeneous polynomials $Q_{p, 2}, Q_{p, 1}$ and every $\lambda \in \mathbb{C}$ there holds the following sharp estimate:

$$
\mu_{\mathcal{G}}\left(Q_{p, 2}-\lambda\left(Q_{p, 1}\right)^{2}\right) \leq 2 \max \{1,|2 \lambda-1|\}
$$

Proof. It is known, that between the functions $p \in \mathcal{C}_{\mathcal{G}}$ and $\varphi \in \mathcal{B}_{\mathcal{G}}(0)$, there holds the following relationship [9]:

$$
p \in \mathcal{C}_{\mathcal{G}} \Longleftrightarrow \frac{p-1}{p+1}=\varphi \in \mathcal{B}_{\mathcal{G}}(0)
$$

Inserting the expansions (3) and (5) of functions into (7), we receive

$$
\sum_{m=1}^{\infty} Q_{p, m}(z)=\left(\sum_{m=1}^{\infty} Q_{\varphi, m}(z)\right)\left(2+\sum_{m=1}^{\infty} Q_{p, m}(z)\right), z \in \mathcal{G} .
$$

Then, comparing the $m$-homogeneous polynomials on both sides of the above equality, we determine the homogeneous polynomials $Q_{\varphi, 1}, Q_{\varphi, 2}$, as follows

$$
\begin{aligned}
Q_{\varphi, 1} & =\frac{1}{2} Q_{p, 1}, \\
Q_{\varphi, 2} & =\frac{1}{2} Q_{p, 2}-\frac{1}{4}\left(Q_{p, 1}\right)^{2} .
\end{aligned}
$$

Putting the above equalities into Theorem 2.1 and using the fact that the mapping $\left(Q_{f, 1}\right)^{2}$ is a 2-homogenous polynomial, we obtain

$$
\frac{1}{2} \mu_{\mathcal{G}}\left[Q_{p, 2}-\frac{1}{2}(1+\gamma)\left(Q_{p, 1}\right)^{2}\right] \leq \max \{1,|\gamma|\}
$$

Denoting

$$
\lambda=\frac{1}{2}(1+\gamma)
$$

we get

$$
\mu_{\mathcal{G}}\left(Q_{p, 2}-\lambda\left(Q_{p, 1}\right)^{2}\right) \leq 2 \max \{1,|2 \lambda-1|\} .
$$

Now, we show the sharpness of the estimate. To do it, let us consider two cases. 
At the beginning, we prove that, in the case

$$
|2 \lambda-1| \geq 1
$$

the equality in (6) is attained by the function $p=\widetilde{p}$ with

$$
\widetilde{p}(z)=\frac{1+I(z)}{1-I(z)}, z \in \mathcal{G} .
$$

Indeed. The function $\widetilde{p}$ belongs to $\mathcal{C}_{\mathcal{G}}$ and $Q_{\widetilde{p}, 1}=2 I, Q_{\widetilde{p}, 2}=2 I^{2}$.

From this, by the case condition for $\lambda$, we have step by step:

$$
\begin{aligned}
\mu_{\mathcal{G}}\left(Q_{\widetilde{p}, 2}-\lambda\left(Q_{\widetilde{p}, 1}\right)^{2}\right) & =\mu_{\mathcal{G}}\left(2 I^{2}-\lambda 4 I^{2}\right)=2|1-2 \lambda| \mu_{\mathcal{G}}\left(I^{2}\right)=2|2 \lambda-1| \\
& =2 \max \{1,|2 \lambda-1|\} .
\end{aligned}
$$

Now, we show that, in the case

$$
|2 \lambda-1|<1
$$

the equality in (6) realizes the function $p=\widehat{p}$, with

$$
\widehat{p}(z)=\frac{1+I^{2}(z)}{1-I^{2}(z)}, z \in \mathcal{G} .
$$

To do it observe that $\hat{p}$ belongs to $\mathcal{C}_{\mathcal{G}}$ and $Q_{\widehat{p}, 1}=0, Q_{\widehat{p}, 2}=2 I^{2}$. From this, by the case condition for $\lambda$, we have:

$$
\mu_{\mathcal{G}}\left(Q_{\widehat{p}, 2}-\lambda\left(Q_{\widehat{p}, 1}\right)^{2}\right)=\mu_{\mathcal{G}}\left(2 I^{2}\right)=2=2 \max \{1,|2 \lambda-1|\} .
$$

This completes the proof.

In the sequel we apply the Fekete-Szegö type result in $\mathcal{C}_{\mathcal{G}}$ to study the family $\mathcal{V}_{\mathcal{G}}$.

We start with the observation that for the transform $\mathcal{L} f$ of the functions $f \in \mathcal{H}_{\mathcal{G}}(1)$, we have

$$
\mathcal{L} f(z)=1+\sum_{m=1}^{\infty} Q_{\mathcal{L} f, m}(z)=1+\sum_{m=1}^{\infty}(m+1) Q_{f, m}(z), z \in \mathcal{G} .
$$

We present the Fekete-Szegö type result in the family $\mathcal{V}_{\mathcal{G}}$ in the following theorem:

Theorem 3. Let $\mathcal{G} \subset \mathbb{C}^{n}$ be a bounded complete n-circular domain and the expansion of the function $f \in \mathcal{V}_{\mathcal{G}}$ into a series of m-homogenous polynomials $Q_{f, m}$ has the form (1), with $Q_{f, 0}=1$. Then for the homogeneous polynomials $Q_{f, 2}, Q_{f, 1}$ and $\eta \in \mathbb{C}$ there holds the following sharp estimate:

$$
\mu_{\mathcal{G}}\left(Q_{f, 2}-\eta\left(Q_{f, 1}\right)^{2}\right) \leq \frac{2}{3} \max \left\{1,\left|\frac{3}{2} \eta-1\right|\right\} .
$$

Proof. Let $f \in \mathcal{V}_{\mathcal{G}}$. Then $p=\mathcal{L} f$ belongs to the family $\mathcal{C}_{\mathcal{G}}$. Inserting into this equality the expansions (5) of functions $p \in \mathcal{C}_{\mathcal{G}}$ and the expansions (8) of $\mathcal{L} f$ of functions $f \in \mathcal{V}_{\mathcal{G}}$, we obtain

$$
1+\sum_{m=1}^{\infty} Q_{p, m}(z)=1+\sum_{m=1}^{\infty}(m+1) Q_{f, m}(z), z \in \mathcal{G} .
$$


Then, comparing the $m$-homogeneous polynomials on both sides of the above equality, we can determine the homogeneous polynomials $Q_{p, 1}, Q_{p, 2}$, as follows

$$
\begin{aligned}
& Q_{p, 1}=2 Q_{f, 1} \\
& Q_{p, 2}=3 Q_{f, 2} .
\end{aligned}
$$

Putting the above equalities into Theorem 2.2 and using the fact that the mapping $\left(Q_{f, 1}\right)^{2}$ is a 2-homogenous polynomial, we obtain

$$
\mu_{\mathcal{G}}\left[3 Q_{f, 2}-4 \lambda\left(Q_{f, 1}\right)^{2}\right] \leq 2 \max \{1,|2 \lambda-1|\}
$$

and consequently

$$
\mu_{\mathcal{G}}\left[Q_{f, 2}-\frac{4}{3} \lambda\left(Q_{f, 1}\right)^{2}\right] \leq \frac{2}{3} \max \{1,|2 \lambda-1|\}
$$

Denoting

$$
\eta=\frac{4}{3} \lambda
$$

we get

$$
\mu_{\mathcal{G}}\left(Q_{f, 2}-\eta\left(Q_{f, 1}\right)^{2}\right) \leq \frac{2}{3} \max \left\{1,\left|\frac{3}{2} \eta-1\right|\right\} .
$$

Now, we will show the sharpnes of the estimates (9).To this aim, we consider two cases.

At the begining, we prove that the equality in (9) holds in the case

$$
\left|\frac{3}{2} \eta-1\right| \geq 1
$$

To do it let us denote by $\mathcal{Z}$ the analytic set $\{z \in \mathcal{G}: I(z)=0\}$. In this case the extremal function has the form

$$
\tilde{f}(z)=\left\{\begin{array}{c}
-1-\frac{2}{I(z)} \log (1-I(z)), \text { for } z \in \mathcal{G} \backslash \mathcal{Z}, \\
1, \text { for } z \in \mathcal{Z}
\end{array},\right.
$$

where the branch of the function $\log (1-\zeta), \zeta \in U$, takes the value 0 at the point $\zeta=0$.

First we observe that $\widetilde{f} \in \mathcal{V}_{\mathcal{G}}$, because $\mathcal{L} \widetilde{f}=\frac{1+I}{1-I} \in \mathcal{C}_{\mathcal{G}}$.

Now we show that $\widetilde{f}$ realizes the equality in the thesis. To do it observe that the power series expansion of the function $\log (1-\zeta), \zeta \in U$, implies the expression

$$
\widetilde{f}(z)=-1+\frac{2}{I(z)}\left(I(z)+\frac{1}{2} I^{2}(z)+\frac{1}{3} I^{3}(z)+\ldots\right), z \in \mathcal{G} .
$$

Thus

$$
\begin{aligned}
& Q_{\widetilde{f}, 1}(z)=I(z) \\
& Q_{\widetilde{f}, 2}(z)=\frac{2}{3} I^{2}(z) .
\end{aligned}
$$


Hence, we have step by step:

$$
\mu_{\mathcal{G}}\left(Q_{\widetilde{f}, 2}-\eta\left(Q_{\widetilde{f}, 1}\right)^{2}\right)=\mu_{\mathcal{G}}\left(\frac{2}{3} I^{2}-\eta I^{2}\right)=\left|\frac{2}{3}-\eta\right| \mu_{\mathcal{G}}\left(I^{2}\right)=\left|\frac{2}{3}-\eta\right|=\frac{2}{3} \max \left\{1,\left|\frac{3}{2} \eta-1\right|\right\} .
$$

Now, we show that, in the case

$$
\left|\frac{3}{2} \eta-1\right|<1
$$

the extremal function has the form

$$
\widehat{f}(z)=\left\{\begin{array}{c}
-1+\log \frac{1+I(z)}{1-I(z)}, \text { for } z \in \mathcal{G} \backslash \mathcal{Z}, \\
1, \text { for } z \in \mathcal{Z}
\end{array},\right.
$$

where the branch of the function $\log (1-\zeta), \zeta \in U$, takes the value 0 at the point $\zeta=0$.

Of course, $\widehat{f} \in \mathcal{V}_{\mathcal{G}}$, because $\mathcal{L} \widehat{f}=\frac{1+I^{2}}{1-I^{2}} \in \mathcal{C}_{\mathcal{G}}$.

Observe that using the power series expansion of the function $\log (1-\zeta), \zeta \in U$, we get the expression

$$
\widehat{f}(z)=-1+\frac{1}{I(z)}\left[2 I(z)+\frac{2}{3} I^{3}(z)+\ldots\right], z \in \mathcal{G}
$$

and consequently

$$
Q_{\widehat{f}, 1}=0, Q_{\widehat{f}, 2}=\frac{2}{3} I^{2}
$$

Therefore, we have step by step

$$
\mu_{\mathcal{G}}\left(Q_{\widehat{f}, 2}-\eta\left(Q_{\widehat{f}, 1}\right)^{2}\right)=\mu_{\mathcal{G}}\left(Q_{\widehat{f}, 2}\right)=\mu_{\mathcal{G}}\left(\frac{2}{3} I^{2}\right)=\frac{2}{3}=\frac{2}{3} \max \left\{1,\left|\frac{3}{2} \eta-1\right|\right\} .
$$

This completes the proof.

\section{Complementary Remarks}

Bavrin [9] declared that every of the estimations (2) is sharp in this sense that there exists an $n$-circular complete bounded domain $\mathcal{G}$ and a function $f$ from appropriate family $\left(f \in \mathcal{B}_{\mathcal{G}}, f \in \mathcal{C}_{\mathcal{G}}, f \in \mathcal{V}_{\mathcal{G}}\right)$ for which the equality in an inequality of (2) holds. Actually we know that the estimations (2) are sharp in the sense that for every domain $\mathcal{G}$ there exists an extremal function in appropriate family which realizes equality in required inequality from (2). Another problem, connected with the above type estimates, is a characterization of the set of all extremal functions. An information in this direction follows from the main result of Reference [12]. Here we present its part connected with the family $\mathcal{C}_{\mathcal{G}}$ (in the term of $\mu_{\mathcal{G}}$-balance of $m$-homogeneous polynomials).

If the function $p$ of the form (5) belongs to $\mathcal{C}_{\mathcal{G}}$, then for every $m \geq 1$

$$
2-\mu_{\mathcal{G}}\left(Q_{p, m}\right) \leq m^{2}\left[2-\mu_{\mathcal{G}}\left(Q_{p, 1}\right)\right] .
$$

Observe that this result implies that the equality $\mu_{\mathcal{G}}\left(Q_{p, 1}\right)=2$ for a function $p \in \mathcal{C}_{\mathcal{G}}$ implies equalities $\mu_{\mathcal{G}}\left(Q_{p, m}\right)=2, m \geq 1$. In others words if a function $p \in \mathcal{C}_{\mathcal{G}}$ is extremal in the estimation (2) for $m=1$, then it is also extremal for each $m \geq 1$. 
Actually, we also have a similar result for the family $\mathcal{V}_{\mathcal{G}}$. More precisely, it is true the following statement. If the function $f$ of the form $(1)$, with $Q_{f, 0}=1$, belongs to $\mathcal{V}_{\mathcal{G}}$, then for every $m \geq 1$

$$
\frac{2}{m+1}-\mu_{\mathcal{G}}\left(Q_{f, m}\right) \leq \frac{2 m^{2}}{m+1}\left[1-\mu_{\mathcal{G}}\left(Q_{f, 1}\right)\right]
$$

To this aim it suffices to recall that, by the assumptions, the function

$$
p(z)=\mathcal{L} f(z)=1+\sum_{m=1}^{\infty}(m+1) Q_{f, m}(z), z \in \mathcal{G},
$$

belongs to the family $\mathcal{C}_{\mathcal{G}}$ and use the previous original inequality in $\mathcal{C}_{\mathcal{G}}$. Therefore, if a function $f \in \mathcal{V}_{\mathcal{G}}$ is extremal in appropriate estimate (2) for $m=1$, that is, if $\mu_{\mathcal{G}}\left(Q_{f, 1}\right)=1$, then it is also extremal in required estimate (2) for each $m \geq 1$, that is, $\mu_{\mathcal{G}}\left(Q_{f, m}\right)=\frac{2}{m+1}$.

We close the paper with a suggestion of characterization of the set of all extremal functions in different estimates of homogeneous polynomials (also of Fekete-Szegö type) in series of functions from subfamilies of the family $\mathcal{H}_{\mathcal{G}}$.

Author Contributions: Investigation, R.D. and P.L. All authors have read and agreed to the published version of the manuscript.

Funding: This research received no external funding.

Conflicts of Interest: The authors declare no conflict of interest.

\section{Reference}

1. Graham, I.G. Kohr, Geometric Function Theory in One and Higher Dimensions; Marcel Dekker, Inc.: New York, NY, USA; Basel, Switzerland, 2003.

2. Kohr, G.; Liczberski, P. Univalent Mappings of Several Complex Variables; Cluj Univ. Press: Cluj-Napoca, Romania, 1998.

3. Miller, S.S.; Mocanu, P.T. Differential Subordinations. Theory and Applications; Marcel Dekker, Inc.: New York, NY, USA; Basel, Switzerland, 2000.

4. Rudin, W. Functional Analysis; McGraw-Hill Inc.: New York, NY, USA, 1991.

5. Temljakov, A. Integral representation of functions of two complex variables. Izv. Acad. Sci. SSSR, Ser. Math. 1957, 21, 89-92.

6. Długosz, R. Embedding theorems for holomorphic functions of several complex variables. J. Appl. Anal. 2013, 19, 153-165. [CrossRef]

7. Długosz, R.; Leś, E. Embedding theorems and extremal problems for holomorphic functions on circular domains of $\mathbb{C}^{n}$. Complex Var. Elliptic Eq. 2014, 59, 883-899. [CrossRef]

8. Les-Bomba, E.; Liczberski, P. New properties of some families of holomorphic functions of several complex variables. Demonstr. Math. 2009, 42, 491-503. [CrossRef]

9. Bavrin, I.I. A class of regular bounded functions in the case of several complex variables and extreme problems in that class. Moskov Obl. Ped. Inst. Moscov 1976, 1-99.

10. Fukui, S. On the estimates of coefficients of analytic functions. Sci. Rep. Tokyo Kyoiku Daigaku Sec. A 1969, 10, 216-218.

11. Higuchi, T. On coefficients of holomorphic functions of several complex variables. Sci. Rep. Tokyo Kyoiku Daigaku 1965, 8, 251-258.

12. Liczberski, P. Extremal problems in certain classes of holomorphic functions of two complex variables. Sci. Bull. Łódź Techn. Univ. Math. 1977, 11, 65-71. 
13. Michiwaki, Y. Note on some coefficients in a starlike functions of two complex variables. Res. Rep. Nagaoka Tech. Coll. 1963, 1, 151-153.

14. Fekete, M.; Szegö, G. Eine Bemerkung über ungerade schlichte Funktionen. J. Lond. Math. Soc. 1933, 8, 85-89. [CrossRef]

15. Keogh, F.R.; Merkes, E.P. A coefficient inequality for certain classes of analytic functions. Proc. Am. Math. Soc. 1969, 20, 8-12. [CrossRef]

16. Gelfer, S.A. On a class of regular functions which ommit any pair w,-w of values. Mat. $S b$ 1946, 19, 33-46.

17. Golusin, G.M. Estimates for analytic functions with bounded mean of the modulus. Trav. Inst. Math. Stekloff 1946, 18, 3-88.

Publisher's Note: MDPI stays neutral with regard to jurisdictional claims in published maps and institutional affiliations.

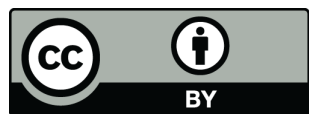

(C) 2020 by the authors. Licensee MDPI, Basel, Switzerland. This article is an open access article distributed under the terms and conditions of the Creative Commons Attribution (CC BY) license (http:/ / creativecommons.org/licenses/by/4.0/). 